\title{
GROWTH CONDITIONS FOR THIN SETS IN VILENKIN GROUPS OF BOUNDED ORDER
}

\author{
D. J. GRUBB
}

(Communicated by J. Marshall Ash)

Abstract. Let $G$ be a Vilenkin group of bounded order and $H_{n}$ a sequence of clopen subgroups of $G$ forming a base at the identity. If $E$ is a subset of $G$, let $N_{n}(E)$ denote the number of cosets of $H_{n}$ which intersect $E$. If

$$
\varliminf \frac{N_{n}(E)}{\log \left[G: H_{n}\right]}<\infty,
$$

then $E$ is a U-set in the group $G$. It is also shown that for $G$ satisfying a growth condition and $\varphi(n) \rightarrow \infty$, there is an M-set, $E$, with

$$
N_{n}(E)=O\left(\varphi(n) \log \left[G: H_{n}\right]\right) \text {. }
$$

Let $G$ be a Vilenkin group, i.e., a compact, totally disconnected, metric group, and let $H_{n}$ be a decreasing sequence of open subgroups of $G$ forming a neighborhood base at the identity of $G$. If $\Gamma$ is the Pontryagin dual of $G$, then for every complex valued function $\left\{c_{\gamma}\right\}$ on $\Gamma$, it is possible to form the "partial sums of the trigonometric series"

$$
S_{n}(c, x)=\sum_{\gamma \in H_{n}^{\perp}} c_{\gamma} \gamma(x) .
$$

We say that a subset $E$ of $G$ is a set of uniqueness (U-set) if the only function $\left\{c_{\gamma}\right\}$ which vanishes at infinity on $\Gamma$ and whose partial sums converge to 0 off $E$ is the zero series. In [1] the author showed that for closed sets this is equivalent to the statement that $E$ supports no pseudofunctions. If a set is not a U-set, it is called an M-set.

In 1972, Kaufman [4] showed in the classical setting that if a subset of the circle intersects $O(\log (1 / \varepsilon))$ intervals of length $\varepsilon$, then that subset is a U-set. The first goal of this paper is to show that an analogous result holds for Vilenkin groups of bounded order.

The second goal of this paper is to obtain a result similar to one of Kahane (see [3]). In Kahane's theorem, it is shown that there are M-sets on the circle intersecting $O(\varphi(\varepsilon) \log (1 / \varepsilon))$ intervals of length $\varepsilon$ as long as $\varphi(\varepsilon) \rightarrow \infty$ as $\varepsilon \rightarrow 0$.

For a subset $E$ of $G$, let $N_{n}(E)$ denote the number of cosets of $H_{n}$ that intersect $E$. 1992.

Received by the editors July 23, 1991 and, in revised form, January 13, 1992 and February 26,

1991 Mathematics Subject Classification. Primary 42C25; Secondary 43A46.

(C) 1993 American Mathematical Society $0002-9939 / 93 \$ 1.00+\$ .25$ per page 
Theorem 1. Let $G$ be a group that is of bounded order. If $E$ is a subset of $G$ with

$$
\varliminf_{\lim } \frac{N_{n}(E)}{\log \left[G: H_{n}\right]}<\infty,
$$

then $E$ is a set of uniqueness.

Proof. Choose $\varepsilon>0$ so that $\gamma \in \Gamma$ and $|\gamma(x)-1|<\varepsilon$ implies $\gamma(x)=1$. This is possible since $G$ is of bounded order. Without loss of generality, we will assume that $N_{n}(E)<C \log \left[G: H_{n}\right]$ for all $n \geq 0$. Now choose $r$ to be an integer such that $r>2 C \log \left(\pi / \sin ^{-1}(\varepsilon / 2)\right)$. For each $n$, let $L_{n}$ be a finite set with $L_{n} H_{n}=E H_{n}$ and with $\operatorname{card}\left(L_{n}\right)<C \log \left[G: H_{n}\right]$. Break $L_{n}$ into $r$ disjoint subsets $L_{j, n}$ of approximately equal size. There are at most $\mathrm{Cr}^{-1} \log \left[G: H_{n}\right]$ elements in each $L_{j, n}$. But

$$
\left(\frac{\pi}{\sin ^{-1}(\varepsilon / 2)}\right)^{C r^{-1} \log \left[G: H_{n}\right]}<\left[G: H_{n}\right]^{1 / 2}<\left[G: H_{n}\right] .
$$

Since there are at most $\pi / \sin ^{-1}(\varepsilon / 2)$ elements of the unit circle such that all distances are more than $\varepsilon$, there are $\gamma_{1}, \gamma_{2} \in H_{n}^{\perp}$ such that

$$
\left|\gamma_{1}(x)-\gamma_{2}(x)\right|<\varepsilon \text { for every } x \in L_{j, n} .
$$

In fact, letting $\gamma=\gamma_{2} \gamma_{1}^{-1}$, we see that the cardianlity of the set

$$
\Gamma_{j, n}=\left\{\gamma \in H_{n}^{\perp}:|\gamma(x)-1|<\varepsilon \text { for all } x \in L_{j, n}\right\}
$$

is at least $\left[G: H_{n}\right]^{1 / 2}$ and so is unbounded for each $j$ as $n \rightarrow \infty$. If $\left(\gamma_{1}, \ldots, \gamma_{p}\right) \in \Gamma_{1, n} \times \cdots \times \Gamma_{r, n}$, then since each $\gamma_{j} \in H_{n}^{\perp}$ and $E H_{n}=L_{n} H_{n}$,

$$
E \subseteq \bigcup_{j=1}^{r}\left\{x:\left|\gamma_{j}(x)-1\right|<\varepsilon\right\} .
$$

By our choice of $\varepsilon$, for such $\left(\gamma_{1}, \ldots, \gamma_{p}\right)$, we have

$$
E \subseteq \bigcup_{j=1}^{r} \operatorname{ker}\left(\gamma_{j}\right)
$$

For each $p \geq 1$, we choose particular $\left(\gamma_{1}, \ldots, \gamma_{r}\right)$ as follows. Choose $n$ large enough so that $\operatorname{card} \Gamma_{j, n} \geq\left(2^{r}-1\right)$ card $H_{p}^{\perp}$.

Pick any $\gamma_{1} \in \Gamma_{1, n}$. If $\gamma_{1}, \ldots, \gamma_{j-1}$ have been chosen, pick

$$
\gamma_{j} \in \Gamma_{j, n} \backslash \bigcup \gamma_{1}^{\varepsilon_{1}} \gamma_{2}^{\varepsilon_{2}} \cdots \gamma_{j-1}^{\varepsilon_{j-1}} H_{p}^{\perp}
$$

where the union is over all sequences $\varepsilon_{1}, \ldots, \varepsilon_{j-1}$ whose terms are all -1 or 0 . Because the cardinality of $\Gamma_{j, n}$ is large, such a choice is possible. The upshot is that no character of the form $\gamma_{1}^{\varepsilon_{1}} \cdots \gamma_{r}^{\varepsilon_{r}}$, where each $\varepsilon_{j}$ is 0 or 1 , can be in $H_{p}^{\perp}$ unless each $\varepsilon_{j}=0$. Now define

$$
f_{p}=\prod_{j=1}^{r}\left(1-\gamma_{j}\right) .
$$


Then $f_{p}=0$ on the neighborhood $E H_{n}$ of $E$ and $\left(1-f_{p}\right)^{\wedge}(\chi)=0$ if $\chi \in H_{p}^{\perp}$. If $S$ is any pseudofunction supported on $E$, we have $S\left(f_{p}\right)=0$, so

$$
|\widehat{S}(1)| \leq\left(2^{r}-1\right) \sup |\widehat{S}(\chi)|
$$

where the supremum is taken over those $\chi \notin H_{p}^{\perp}$. Since $r$ is fixed and the supremum goes to 0 as $p \rightarrow \infty, \widehat{S}(1)=0$. By a standard argument, $S=0$. Thus $E$ is a U-set.

It was previously known that if $N_{n}(E)=o\left(\log \left[G: H_{n}\right]\right)$, then $E$ is a Dirichlet set and hence a strong U-set [2].

Now we turn to the question of the converse: how slow can $N_{n}(E)$ grow for an M-set? For the classical case, [3] shows that if $\varphi(\varepsilon) \rightarrow \infty$ as $\varepsilon \rightarrow 0$ there are sets in the circle group such that $N_{\varepsilon}(E)=O(\varphi(\varepsilon) \log (1 / \varepsilon))$ and yet $E$ fails to be a U-set. To get this result, Kahane used the properties of Brownian motion. For the corresponding result in the current setting, we have to produce an analog of Brownian motion on Vilenkin groups. I would like to thank Robert Kaufman for his suggestions at this point.

Let $\xi_{x H_{n}}$ be a random point with values in $H_{n}$ which is uniformly distributed with respect to normalized Haar measure on $H_{n}$. We will assume that different $\xi_{x H_{k}}$ are independent random points. Thus, if $\gamma \in \Gamma$, we have

$$
E\left[\gamma\left(\xi_{x H_{n}}\right)\right]= \begin{cases}1 & \text { if } \gamma \in H_{n}^{\perp}, \\ 0 & \text { otherwise. }\end{cases}
$$

Notice that there is a separate random point for each coset of each $H_{n}$. We now define a random function on $G$ by

$$
\psi(x)=\sum_{n=0}^{\infty} \xi_{x H_{n}} .
$$

Notice that this sum converges for every point in $G$ and that for two points $x, y \in G$, if $x H_{n}=y H_{n}$ then $\psi(x) H_{n}=\psi(y) H_{n}$. Thus $\psi$ enjoys a type of Lipschitz continuity. Thus we have for a subset $E$ of $G$,

$$
N_{n}(\psi(E)) \leq N_{n}(E) .
$$

Now we present a converse to Theorem 1.

Theorem 2. Let $G$ be a Vilenkin group. Assume that there is a constant $C$ such that

$$
\sum_{n=0}^{\infty} \frac{\left[G: H_{n+1}\right]}{\left[G: H_{n}\right]^{C}}<\infty .
$$

Let $\varphi(n) \uparrow \infty$ as $n \rightarrow \infty$. Then there exists an $M$-set $E$ in $G$ such that

$$
N_{n}(E)=O\left(\varphi(n) \log \left[G: H_{n}\right]\right) \text {. }
$$

Proof. Let $\theta(n)=\varphi(n) \log \left[G: H_{n}\right]$. Let $k_{n}$ be integers such that

$$
\theta(n) \leq k_{1} k_{2} \cdots k_{n} \leq 2 \theta(n) .
$$

Pick $k_{n}$ points of $H_{n}$ in distinct cosets of $H_{n+1}$ and let $\mu_{n}$ be a discrete measure on these points giving equal measure to each of them. Let $\mu=*_{n=1}^{\infty} \mu_{n}$. Then $\mu$ is a measure supported on a set $E$ with

$$
N_{n}(E) \leq 2 \theta(n) \text { and } \mu\left(x H_{n}\right) \leq 1 / \theta(n) \text { for all } n \geq 0 .
$$


Now consider the random measure $\nu$ where

$$
\int f d \nu=\int f \circ \psi d \mu
$$

For $\gamma \in H_{n+1}^{\perp} \backslash H_{n}^{\perp}$, we have

$$
\begin{aligned}
\hat{\nu}(\gamma) & =\int_{G} \gamma \circ \psi d \mu=\sum_{x H_{n}} \int_{x H_{n}} \gamma \circ \psi d \mu=\sum_{x H_{n}} \int_{x H_{n}} \prod_{k=0}^{\infty} \gamma\left(\xi_{y H_{k}}\right) d \mu(y) \\
& =\sum_{x H_{n}} \int_{x H_{n}} \prod_{k=0}^{n} \gamma\left(\xi_{x H_{k}}\right) d \mu(y)=\sum_{x H_{n}} \mu\left(x H_{n}\right) \prod_{k=0}^{n} \gamma\left(\xi_{x H_{k}}\right) .
\end{aligned}
$$

Notice that for $y \in x H_{n}$, it follows that $\xi_{x H_{k}}=\xi_{y H_{k}}$ for all $k \leq n$. For each coset $x H_{n}$, we introduce the random variable

$$
Y_{x H_{n}}=\mu\left(x H_{n}\right) \prod_{k=0}^{n} \gamma\left(\xi_{x H_{k}}\right) .
$$

Then $\left|Y_{x H_{n}}\right|=\mu\left(x H_{n}\right)$, and by taking conditional expectations with respect to all the $\xi_{x H_{n}}$ for this fixed $n$, we have that the $Y_{x H_{n}}$ are conditionally independent and

$$
E\left[Y_{x H_{n}} \mid\left\{\xi_{x H_{n}}: x \in G\right\}\right]=0 .
$$

Thus, using a sub-Gaussian inequality, we have for real $t$,

$$
\begin{aligned}
& E\left[\exp (t \operatorname{Re} \hat{\nu}(\gamma)) \mid\left\{\xi_{x H_{n}}: x \in G\right\}\right] \\
& \quad=E\left[\exp \left(\sum_{x H_{n}} t \operatorname{Re} Y_{x H_{n}}\right) \mid\left\{\xi_{x H_{n}}: x \in G\right\}\right] \\
& \quad=\prod_{x H_{n}} E\left[\exp \left(t \operatorname{Re} Y_{x H_{n}}\right) \mid\left\{\xi_{x H_{n}}: x \in G\right\}\right] \leq \prod_{x H_{n}} \exp \left(t^{2} \mu\left(x H_{n}\right)^{2}\right) \\
& \quad=\exp \left(t^{2} \sum_{x H_{n}} \mu\left(x H_{n}\right)^{2}\right) \leq \exp \left(\frac{t^{2}}{\theta(n)}\right) .
\end{aligned}
$$

Now we have

$$
E[\exp (t \operatorname{Re} \hat{\nu}(\gamma))] \leq \exp \left(\frac{t^{2}}{\theta(n)}\right)
$$

A similar expression holds for $\operatorname{Im} \hat{\nu}(\gamma)$. Recalling the definition of $\theta(n)$, we see

$$
\begin{aligned}
P\left[\operatorname{Re} \hat{\nu}(\gamma)>\varphi(n)^{-1 / 3}\right] & \leq \exp \left(-\frac{1}{4} \varphi(n)^{1 / 3} \log \left[G: H_{n}\right]\right) \\
& \leq \frac{1}{\left[G: H_{n}\right]^{\varphi(n)^{1 / 3} / 4}} .
\end{aligned}
$$

This holds for each $\gamma \in H_{n+1}^{\perp} \backslash H_{n}^{\perp}$, so

$$
P\left[\exists \gamma \in H_{n+1}^{\perp} \backslash H_{n}^{\perp} \text { such that } \operatorname{Re} \hat{\nu}(\gamma)>\varphi(n)^{-1 / 3}\right]<\frac{\left[G: H_{n+1}\right]}{\left[G: H_{n}\right]^{\varphi(n)^{1 / 3} / 4}} .
$$


Since similar expressions hold for $-\operatorname{Re} \hat{\nu}(\gamma)$ and $\operatorname{Im} \hat{\nu}(\gamma)$ in place of $\operatorname{Re} \hat{\nu}(\gamma)$, and since $\varphi(n) \rightarrow \infty$, an application of the Borel-Cantelli Lemma along with (8) shows that $\lim \hat{\nu}(\gamma)=0$ almost surely.

Notice that all Vilenkin groups of bounded order satisfy the condition in (8). In fact, any group with growth like $\exp (\exp (n))$ satisfies this condition.

Using the methods above, we may prove the following result.

Theorem 3. Let $G$ be a Vilenkin group satisfying the condition in (8). Given $\delta>0$ there is a subset $E$ of $G$ such that $N_{n}(E)=O\left(\log \left[G: H_{n}\right]\right)$ and $a$ measure $\nu$ supported on $E$ with $\varlimsup \lim |\hat{\nu}(\gamma)| \leq \delta$, where the limit is taken as $\gamma \rightarrow \infty$ in $\Gamma$. By Theorem 2 of [2], we see that $E$ is not a Dirichlet set.

Proof. In the previous proof, simply let $\varphi(n)$ be a constant where $\varphi(n)^{1 / 3}>4 C$ and $\varphi(n)^{-1 / 3}<\delta$.

\section{ACKNOWLEDGMENT}

I would like to thank Robert Kaufman of the University of Illinois for suggestions leading to substantial improvements in Theorems 2 and 3.

\section{REFERENCES}

1. D. J. Grubb, U-sets in compact, 0-dimensional, metric groups, Canad. Math. Bull. 32 (1989), 149-155.

2. __ Dirichlet sets in Vilenkin groups, Acta Math. Hungar. (to appear).

3. J. P. Kahane, A metric condition for a closed circular set to be a set of uniqueness, J. Approx. Theory 2 (1969), 233-246.

4. Robert Kaufman, Kronecker sets and metric properties of $M_{0}$-sets, Proc. Amer. Math. Soc. 36 (1972), 519-524.

Department of Mathematics, Kansas State University, Manhattan, Kansas 66506 Current address: Department of Mathematical Sciences, Northern Illinois University, DeKalb, Illinois 60115

E-mail address: grubb@math.niu.edu 